\title{
Prevalence of breast tuberculosis: Retrospective analysis of 65 patients attending a tertiary hospital in Durban, South Africa
}

\author{
D P Ramaema, ${ }^{1}$ FCRad (Diag) SA; I Buccimazza, ${ }^{2}$ FCS (SA), FACS; R J Hift, ${ }^{3}$ MMed (Med), PhD, FCRP, FCP (SA) \\ ${ }^{1}$ Division of Radiation Medicine (Radiology), School of Clinical Medicine, College of Health Sciences, Nelson R Mandela School of Medicine, \\ University of KwaZulu-Natal, Durban, South Africa \\ ${ }^{2}$ Division of General Surgery, School of Clinical Medicine, College of Health Sciences, Nelson R Mandela School of Medicine, University of \\ KwaZulu-Natal, Durban, South Africa \\ ${ }^{3}$ Division of Medicine, School of Clinical Medicine, College of Health Sciences, Nelson R Mandela School of Medicine, University of KwaZulu- \\ Natal, Durban, South Africa
}

Corresponding author: D P Ramaema (ramaema@ukzn.ac.za)

\begin{abstract}
Background. Breast tuberculosis (BTB) is uncommon, but not rare. Knowledge of the ways in which it can present can prevent unnecessary invasive procedures and delay in diagnosis.

Objectives. To describe the clinical and radiological findings in patients with BTB, including evaluation of current treatment methods. Methods. We retrospectively analysed 65 patients diagnosed with BTB at Addington and King Edward VIII hospitals, Durban, South Africa, between 2000 and 2013. Demographic, clinical and radiological findings and treatment outcomes were noted.

Results. A total of 11092 patients underwent breast investigations between 2009 and 2013, with a prevalence of BTB for the period of $0.3 \%$ (30 patients). Of the 65 patients diagnosed between 2000 and 2013, 64 were female (98.5\%) and one was male (1.5\%). The age range was 23 69 years (mean 38.5$)$. The most common mammographic pattern was density $(39.4 \%)$ and the least common a mass $(6.1 \%)$. Isolated axillary lymphadenitis was found in $12.1 \%$. Abscess was the commonest ultrasound pattern (39.0\%). Of the 47 patients with a known history of pulmonary tuberculosis (TB), $68.1 \%(n=32)$ did not have radiological evidence of previous or concurrent pulmonary TB, nor was there evidence of TB elsewhere. Of 47 patients with known HIV status, 34 were HIV-positive. Fine-needle aspiration cytology had sensitivity of only $28 \%$ compared with $94 \%$ for histology. Of those treated, $72.7 \%$ obtained full resolution following 9 months of TB treatment; $25.0 \%$ did not complete treatment, and $2.3 \%(n=1)$ died while on treatment. Follow-up data on relapse rates after treatment completion and disease resolution are scanty.

Conclusion. Understanding and being aware of the various presentations of BTB make it possible to treat most patients successfully.

S Afr Med J 2015;105(10):866-869. DOI:10.7196/SAMJnew.7704
\end{abstract}

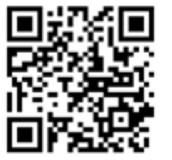

It is estimated that over 1 billion people worldwide have tuberculosis (TB). ${ }^{[1]}$ However, breast tuberculosis (BTB) is a rare condition. The incidence has been reported as $<0.1 \%$ of all surgical breast lesions seen in developed countries and $3-4.5 \%$ of breast lesions in countries where BTB is endemic, ${ }^{[2,3]}$ although even in this setting, carcinoma is far more commonly encountered. ${ }^{[4]}$ While BTB is considered a manifestation of extrapulmonary tuberculosis (EPTB), it represents only about $0.1 \%$ of the disease burden of EPTB. However, there has been a resurgence of EPTB as a result of HIV, with estimated incidences as high as $50 \%$ in countries where HIV infection is endemic..$^{[5]}$

The right and left breasts are equally affected by BTB, which may be bilateral in $\sim 3 \%$ of cases. ${ }^{[6]} \mathrm{Up}$ to $70 \%$ of patients may have associated axillary lymphadenopathy, often with visible axillary swelling. ${ }^{[7]}$ Constitutional symptoms are unusual in the absence of systemic and particularly pulmonary TB. ${ }^{[4]}$

No clinical or radiological features are absolutely diagnostic of BTB. The diagnosis is usually readily confirmed by biopsy with histological examination or fine-needle aspiration and staining for acid-fast bacilli (AFB), mycobacterial culture or polymerase chain reaction (PCR) testing. While the main differential diagnosis is carcinoma, other considerations are idiopathic granulomatous mastitis and fungal infections. Most patients respond to standard antiTB therapy (ATT) ${ }^{[8]}$ It has been suggested that patients in endemic areas who have a breast mass with granulomatous inflammation on histological examination should receive ATT, even if culture results are negative for $\mathrm{TB} .{ }^{[9]}$ There have been reports that sinuses, fistulas and deformities may occasionally require primary localised excision or simple mastectomy, but this is not standard care. ${ }^{[4]}$

\section{Objectives}

To identify the various radiological patterns of BTB, and to assess available treatment options and response to treatment.

\section{Methods}

We identified 64 patients with a histological or cytological confirmed diagnosis of BTB. One patient with discharging axillary nodes and breast oedema was included even though cytological findings were not confirmatory; 65 patients were therefore included in the data analysis. Patients were diagnosed between 2000 and 2013. Data were collected from Addington ( $n=60$ patients) and King Edward VIII hospitals $(n=5)$, Durban, South Africa. The study was approved by the Bioethics Research Committee at the University of KwaZuluNatal, Durban (Reference No. BF213/13). A retrospective chart and radiology review focused on demographic, clinical, mammographic and ultrasound patterns and diagnostic and treatment methods.

Mammograms (mediolateral oblique (MLO) and craniocaudal views) were done using a standard Mecatronica analogue mammographic unit ( \pm digital processing). Ultrasound images 
were obtained on the various standard units using a $7.5-12.5 \mathrm{MHz}$ linear probe. Mammographic patterns were classified as follows: asymmetrical density, asymmetrical density with axillary nodes, inflammation with axillary nodes, mass, mass with axillary nodes, nodes only, and normal. Ultrasound patterns were classified as abscess, abscess with axillary nodes, mass, mass with axillary nodes, oedema,

\begin{tabular}{|c|c|}
\hline Characteristics & \\
\hline \multicolumn{2}{|l|}{ Gender, $n(\%)$} \\
\hline Female & $64(98.5)$ \\
\hline Male & $1(1.5)$ \\
\hline \multicolumn{2}{|c|}{ Age at presentation (years) } \\
\hline Range & $23-69$ \\
\hline Mean (SD) & $38.5(10.3)$ \\
\hline \multicolumn{2}{|l|}{ Side, $n(\%)$} \\
\hline Left & $30(46.2)$ \\
\hline Right & $33(50.8)$ \\
\hline Unknown & $2(3.1)$ \\
\hline \multicolumn{2}{|l|}{ Axillary nodes, $n(\%)$} \\
\hline Yes & $21(33.9)$ \\
\hline No & $41(66.1)$ \\
\hline \multicolumn{2}{|c|}{ Clinical presentation, $n(\%)$} \\
\hline Abscess & $2(3.1)$ \\
\hline Adenitis + oedema & $16(24.6)$ \\
\hline Mass (nodular) form & $17(26.2)$ \\
\hline $\begin{array}{l}\text { Inflammatory mass } \\
\text { (disseminated) }\end{array}$ & $26(40.0)$ \\
\hline Occult mass & $1(1.5)$ \\
\hline Unknown & $3(4.6)$ \\
\hline $\mathrm{SD}=$ standard deviation & \\
\hline
\end{tabular}

oedema with nodes, thickening, nodes only, and normal.

Histological and microbiological diagnosis was done by obtaining core biopsy samples using a 14-gauge needle. Cytological diagnosis was done by FNA biopsy. Lesion localisation was either achieved by palpation or ultrasound guided.

Descriptive statistics using proportions were used to analyse results.

\section{Results}

Sixty-four records of patients with pathology-proven BTB and one with strong clinical but inconclusive cytological findings were identified during the period 2000 2013. Accurate clinic attendance records were available for the years 2009 - 2013, and during this 5-year period a total of 11092 patients underwent breast investigations and 30 were diagnosed with BTB (prevalence for the period $0.3 \%$ ). Demographics and

Table 2. Associated clinical features

\begin{tabular}{ll}
\hline Characteristics & $\boldsymbol{n}(\%)$ \\
\hline $\begin{array}{l}\text { History of pulmonary TB } \\
(N=47)\end{array}$ & \\
$\quad$ CXR normal & $32(49.2)$ \\
CXR abnormal & $15(23.1)$ \\
Pregnant/lactating assessed & \\
$(N=65)$ & \\
$\quad$ No & $64(98.5)$ \\
$\quad$ Yes & $1(1.5)$ \\
HIV status $(N=47)$ & $12(18.5)$ \\
Negative & $34(52.3)$ \\
Positive & $1(1.5)$ \\
Refused testing & \\
CXR = chest radiograph.
\end{tabular}

clinical presentation are described in Table 1. The age range was 23 - 69 years (mean 38.5 years). Of the 65 patients, 26 presented with an inflammatory mass or disseminated form of BTB. Other associated clinical features are set out in Table 2 . Of the 65 patients, only one was lactating. HIV status was known in 47 patients, of whom 34 were HIV-positive. While 47 patients had a history of previous pulmonary $\mathrm{TB}$, only 15 had radiological evidence of either previous or concurrent pulmonary TB.

Radiological features on mammograms and ultrasound scans are summarised in Fig. 1 ( $a$ and b, respectively). Of the 33 patients who had mammograms, 16 demonstrated ipsilateral or bilateral axillary lymphadenopathy. A density pattern was seen in 13 cases. Ultrasound scans were done in 41 patients. The commonest pattern was that of an abscess (16 cases, 39.0\%).

Sensitivity for fine-needle aspiration cytology (FNAC) was $28 \%$ as opposed to 94\% for histology. AFB were seen in $10.3 \%$ of the FNAC specimens and $29.7 \%$ of the histology specimens. Of the 65 patients, 59 received medical treatment, 3 received surgical intervention, and 3 were lost to follow-up after diagnosis. Of those who were treated, $72.7 \%$ obtained full resolution, $25.0 \%$ did not complete treatment, and $2.3 \%$ died $(n=1)$.

\section{Discussion}

\section{Clinical and radiological findings}

Inflammatory/disseminated

This pattern was the most common clinical presentation of BTB. However, among patients who had mammograms, this pattern was seen in $21.2 \%$ (Fig. 2, a). The mammogram typically demonstrated nonspecific diffuse stromal thickening. We noted that all cases with an inflammatory/disseminated form also

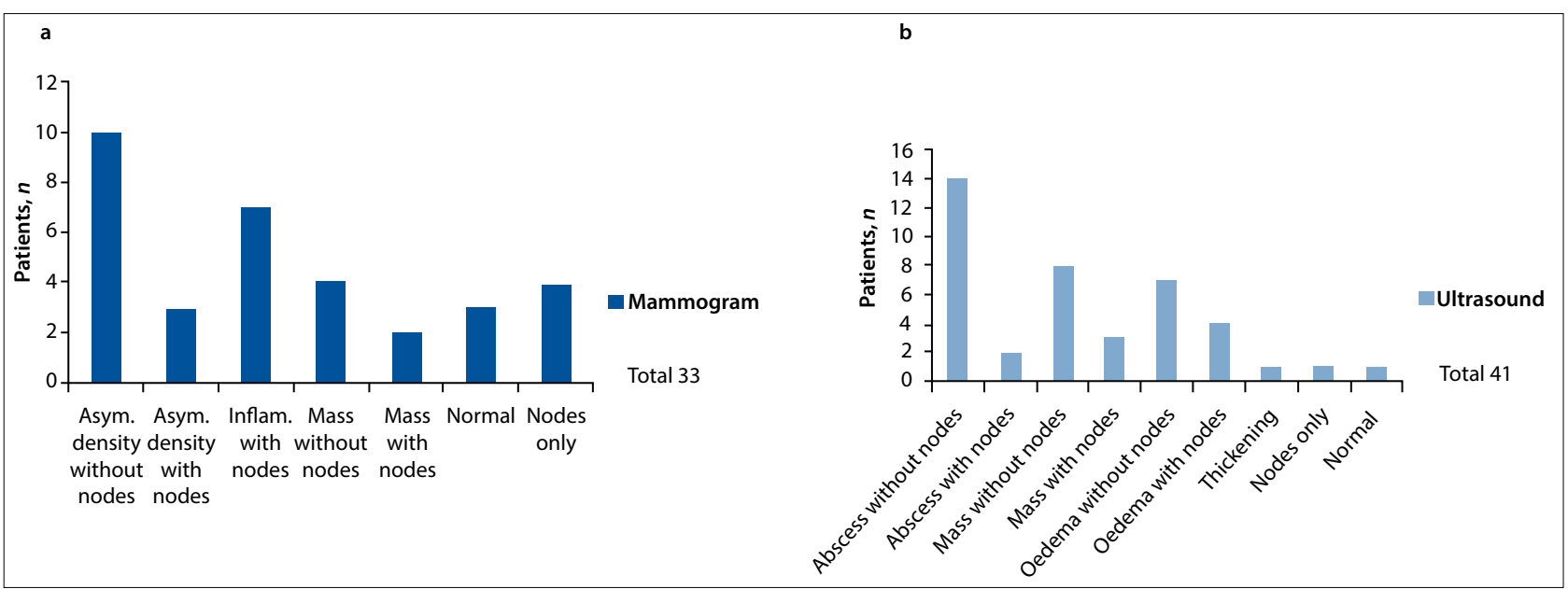

Fig. 1. (a) Of 33 patients who had mammograms, 16 demonstrated ipsilateral or bilateral axillary lymphadenopathy. An asymmetrical density pattern was seen in 13 cases. (b) Ultrasound scans were performed in 41 patients. The commonest pattern was an abscess variety in (16 cases, $39 \%$ ). 
had nodes. This supports the hypothesis that this form may be due to either reactive oedema or retrograde spread from the axillary lymph nodes. ${ }^{[10]}$ To date, no study has specifically determined whether AFB are present in the oedematous or diffusely inflamed breast. Because of the appearances, it has been suggested that this in fact represents the clinical spectrum from inflammatory mass/disseminated to oedema. The major differential diagnosis of this form is inflammatory breast cancer. However, the combination of an inflammatory breast lesion and sinuses or fistulas favours BTB rather than cancer. ${ }^{[3]}$ On the ultrasound scan, some disseminated forms appeared as diffuse trabecular thickening and oedema (Fig. 2, c), whereas others demonstrated a diffuse inflammatory mass.

\section{Oedema with adenitis}

The clinical presentation of oedema was noted in $24.6 \%$ of cases, and an ultrasound pattern of oedema was seen in 26.9\% $(n=11)$. In contrast to the disseminated/inflammatory form, where all had associated axillary lymph nodes, seven patients in this group (17.1\%) did not have associated lymphadenopathy. This pattern can be found in other forms of mastitis.

\section{Abscess variety}

Even though clinical evaluation classified an abscess variety of BTB in only $3.1 \%$, this was the most common pattern on ultrasound, seen in $39.0 \%(n=18)$, with or without adenitis. Ultrasound scans demonstrated complex solid-cystic heterogeneous hypoechoic masses and fluid collections. In our study, asymmetrical density was the commonest mammographic pattern $(39.4 \%, n=13)$ associated with this classification. Three patients only had ultrasound diagnosis. The differential diagnosis was a pyogenic abscess, and some of our patients had in fact received treatment with antibiotics; it was only after lack of response to this that the diagnosis of tubercular abscess was considered. It is therefore important to obtain samples specifically for $\mathrm{TB}$ analysis in all cases of non-lactational breast abscess, even if there is no history of TB elsewhere.

\section{Nodular (focal mass)}

Clinically this form of BTB presented as a focal mass. On the mammograms the focal mass opacities had variable outlines, some having smooth margins while others were irregular. Some were

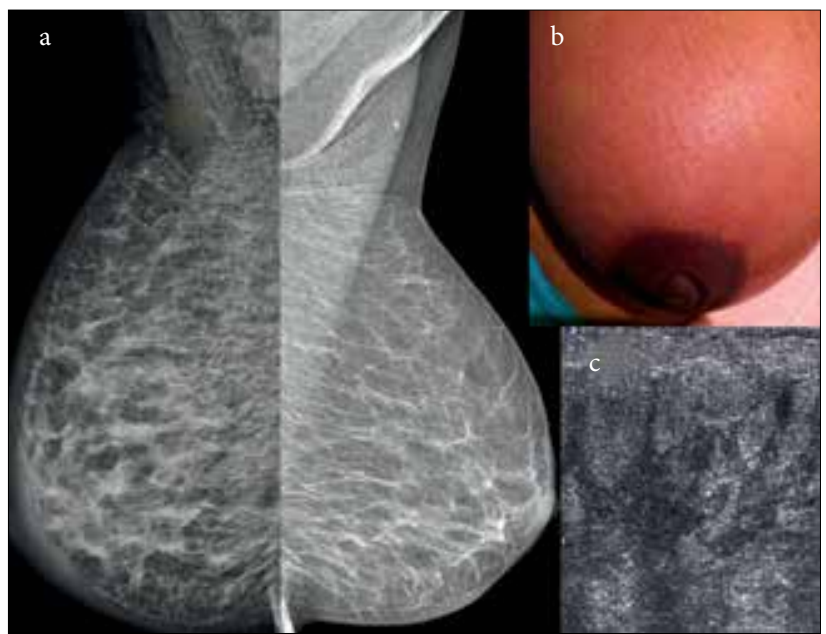

Fig. 2. Inflammatory/disseminated BTB, images taken from various patients. (a) mammogram, MLO views. The right breast is enlarged. Diffuse stromal thickening and skin oedema is evident. (b) Photograph of the left breast, showing inflammation of the skin. (c) Ultrasound images showing diffuse trabecular thickening with oedema as hypoechoic bands in between hyperechoic fibrofatty tissue. single mass lesions, and others were multiple (Fig. 3). This was the third most common mammographic pattern, seen in $18.2 \%$ of patients. On ultrasound this form appeared as a hypoechoic solid or heterogeneous mass with a variable outline ranging from welldefined round to irregular. Lesion size based on ultrasound ranged from $0.9 \mathrm{~cm}$ to $6.1 \mathrm{~cm}$. The differential diagnosis of this form can range from benign fibroadenoma to breast cancer, depending on the lesion character on imaging. We did not find microcalcification to be a feature in our study.

\section{Tuberculous lymphadenitis}

Although isolated lymphadenitis was not reported on clinical presentation, it was seen in $12.1 \%$ of mammograms $(n=4)$ and $2.4 \%$ of ultrasound scans $(n=1)$. Nodes were intramammary, axillary, or both. Clinically, large nodes can extend to the skin surface to form discharging sinuses (Fig. 4, a). The intramammary nodes appeared as well-defined round, dense masses on the mammogram. These can be confused with a breast mass when they are solitary. Radiological differentiation of tuberculous axillary nodes from metastatic nodes

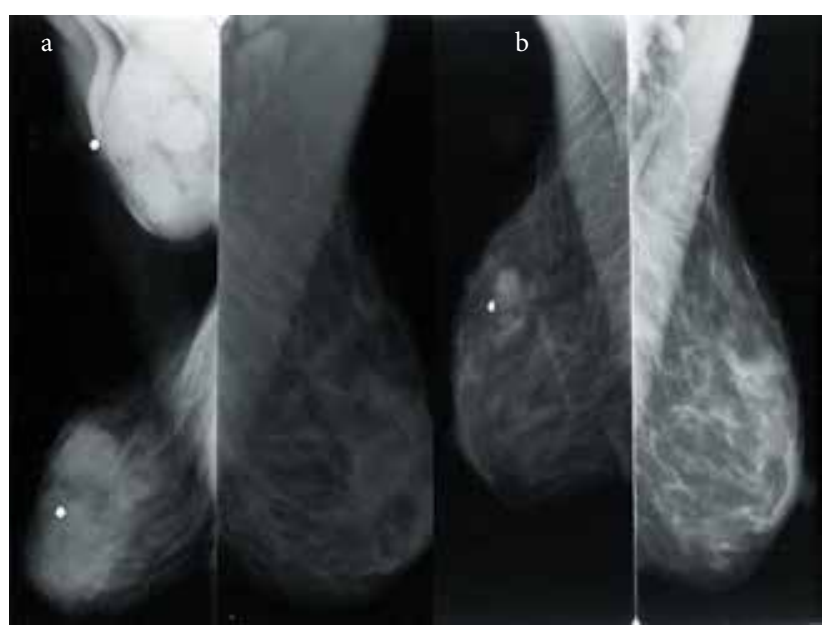

Fig. 3. Nodular form of BTB. Mammogram, MLO views. (a) At presentation - multiple rounded mass opacities in the right breast, and enlarged dense right axillary nodes. (b) After 4 months of treatment with ATT, one small round mass opacity remains in the upper part.

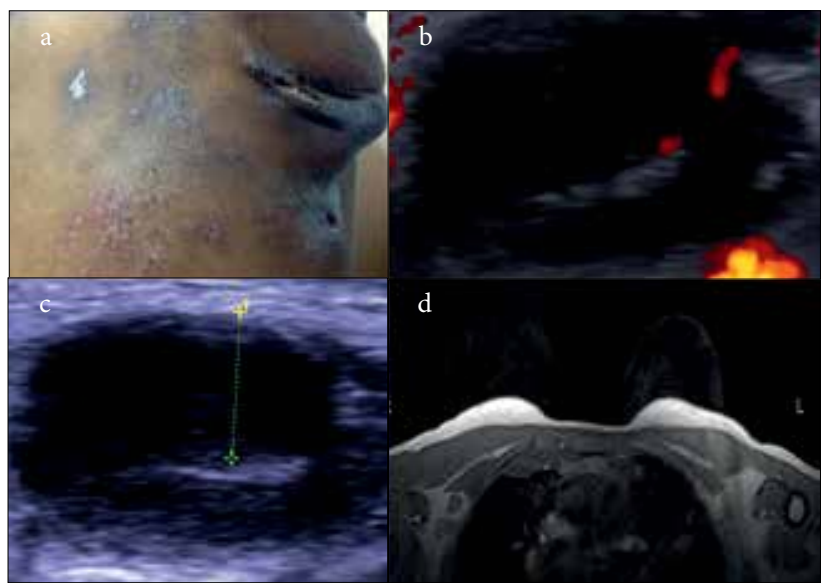

Fig. 4. Tuberculous lymphadenitis. (a) Left axilla, enlarged nodes discharging onto skin sinuses. (b) Ultrasound Doppler scan showing hilum vessel and thick cortex. (c) Ultrasound scan showing node with thick $7 \mathrm{~mm}$ cortex (callipers). (d) Breast MRI T1 post-contrast axial image. Right axillary lymph nodes have peripheral rim enhancement and central low signal (PRECLO). 
remains difficult. In our series, ultrasound scanning of axillary nodes demonstrated oval, hypoechoic solid masses with thick cortices and loss of normal fatty hilum (Fig. $4, \mathrm{~b}$ and c). This appearance cannot be differentiated from that of a malignant or metastatic lymph node. Post-contrast magnetic resonance imaging (MRI) showed peripheral enhancement with a central low signal (Fig. 4, d). Another author described nodes as having peripheral enhancement with central low attenuation (PRECLO) on positive emission tomography with fluorodeoxyglucose integrated with computed tomography $\left({ }^{18} \mathrm{~F}-\mathrm{FDG}\right.$ $\mathrm{PET} / \mathrm{CT}){ }^{[11]}$

\section{Pathological findings}

We found FNAC to be only $28 \%$ sensitive, whereas the sensitivity of histopathology was $94 \%$. Necrotising granulomatous inflammation was seen in histological specimens. In support of the literature, the yield of AFB in our study was low, being positive in $10.3 \%$ of cytology and $29.6 \%$ of histology specimens. In one case, AFB were detected histologically by PCR testing. Harris et al. ${ }^{[12]}$ reported a $2 \%$ rate of positive Ziehl-Neelsen smears for AFB from tuberculous breast abscess fluid. Furthermore, an AFBpositive smear is not always adequate for a definitive diagnosis of Mycobacterium tuberculosis, as it should be differentiated from other Mycobacterium species.

\section{Treatment}

Of the 65 patients, 59 received medical treatment comprising 9 months of standard ATT, 3 received both medical and surgical intervention involving abscess drainage, and 3 were lost to followup and were never treated following diagnosis. Of those who were treated, $72.7 \%$ obtained full clinical resolution, $25.0 \%$ did not complete treatment, and one patient died. Response to treatment was assessed by clinical examination and repeated radiological investigations (ultrasound, mammography or both). Although there are no documented criteria for radiological response, drug resistance may be suspected when lesions fail to reduce in size or change their appearance on ultrasound or mammography. Ultrasound is especially useful in monitoring the abscess form of BTB, to detect residual fluid collections. In single studies, both MRI ${ }^{[13]}$ and ${ }^{18} \mathrm{~F}-\mathrm{FDG}$ PET/CT ${ }^{[11]}$ have shown promise as tools for monitoring response.

\section{Risk factors}

Our data demonstrate the varied spectrum of BTB presentation, both clinical and radiological, as well as good outcome with medical treatment. Furthermore, the study illustrates some association of BTB with HIV. Although most patients with BTB are females, male patients are occasionally affected. Our study supports this, with only one of 65 patients (1.5\%) being male. The mean age of the patients in our study was 38.5 years, with a range of $23-69$ years, demonstrating that BTB is not limited to females of reproductive age, as has been reported in the literature. ${ }^{[14]}$ Several risk factors for BTB have been described, including lactation, multiparity and HIV/ AIDS. ${ }^{[12]}$ We found only one patient (1.5\%) to be lactating. HIV status was known in 47 of our patients, of whom $72.3 \%(n=34)$ were HIV-positive. Although HIV infection has been associated with an increased incidence of EPTB, BTB still remains rare. BTB has been classified as primary or secondary. Whereas most studies report that the primary form of BTB is rare, ${ }^{[9]}$ we found the opposite. A history of pulmonary TB was obtained from 47 patients, of whom $68.1 \%(n=32)$ had a normal chest radiograph and no evidence of pulmonary or concurrent TB or of TB elsewhere in the body. This finding suggests that half of our cohort had the primary form of BTB.

\section{Study limitations}

Limitations due to the retrospective nature of the study include incomplete data on follow-up after treatment completion, even though patients were scheduled for further visits.

\section{Conclusions}

Our findings demonstrate the varied clinical and radiological presentations of BTB. Early diagnosis can lead to full response following treatment with standard ATT, resulting in avoidance of more invasive surgical treatment which can potentially cause breast disfigurement. Clinicians need to be aware of these patterns and to have a high index of suspicion in order to prevent misdiagnosis and inappropriate management.

Sources of support. The University of KwaZulu-Natal (UKZN)'s Medical Education Partnership Initiative (MEPI) Enhancing Training, Research and Education (ENTRÉE) programme (Grant No. 5R24TW008863) provided seed funding and support for the development of this study. MEPI is a National Institutes of Health/PEPFAR-funded grant awarded to UKZN in 2010 that aims to develop or expand and enhance models of medical education in sub-Saharan Africa.

Acknowledgements. Part of the material from this article was presented at the European Congress of Radiology (ECR 2015) as the Electronic Poster Online Submission (EPOS). ${ }^{[15]}$ The authors thank the staff at the breast units at Addington and King Edward VIII hospitals, who facilitated access to radiological and clinical information on study participants.

Conflict of interest. Some of the material in this article was presented at ECR 2015 as the EPOS. ${ }^{[15}$

\section{References}

1. Gupta R, Singal RP, Gupta A, Singal S, Shahi SR, Singal R. Primary tubercular abscess of the breast - an unusual entity. J Med Life 2012;5(1):98-100.

2. Meerkotter D, Spiegel K, Page-Shipp LS. Imaging of tuberculosis of the breast: 21 cases and a review of the literature. I Med Imaging Radiat Oncol 2011:55(5):453-460. [http://dx.doi.org/10.1111/j.17549485.2011.02306.x]

3. Tewari M, Shukla SH. Breast tuberculosis: Diagnosis, clinical features and management. Indian J Med Res 2005;122(2):103-110.

Mehta G, Mittal A, Verma S. Breast tuberculosis - clinical spectrum and management. Indian J Sur 2010;72(6):433-437. [http://dx.doi.org/10.1007/s12262-010-0166-5] Akbulut S, Sogutcu N, Yagmur Y. Coexistence of breast cancer and tuberculosis in axillary lymph
nodes: A case report and literature review. Breast Cancer Res Treat 2011;130(3):1037-1042. [http:// dx.doi.org/10.1007/s10549-011-1634-8]

6. Baslaim MM, Al-Amoudi SA, Al-Ghamdi MA, Ashour AS, Al-Numani TS. Case report: Breast cance associated with contralateral tuberculosis of axillary lymph nodes. World J Surg Oncol 2013;11:43. [http://dx.doi.org/10.1186/1477-7819-11-43]

7. Kumar M, Chand G, Nag VL, et al. Breast tuberculosis in immunocompetent patients at tertiary care center: A case series. J Res Med Sci 2012;17(2):199-202.

Tanrikulu AC, Abakay A, Abakay O, Kapan M. Breast tuberculosis in southeast Turkey: Report of 27 cases. Breast Care (Basel) 2010;5(3):154-157. [http://dx.doi.org/10.1159/000314267]

Shushtari MHS, Alavi SM, Talaeizadeh A. Breast tuberculosis: Report of nine cases of extra pulmonary tuberculosis with breast mass. Pak J Med Sci 2011;27(3):582-585.

10. Oh KK. Kim JH Kook SH. Im of tur Radiol 1998:8(8):14751480. [http://dx.doi.org/10.1007/s003300050578]

11. Sathekge M, Maes A, D'Asseler Y, Vorster M, Gongxeka H, van de Wiele C. Tuberculous lymphadenitis: Sathekge M, Maes A, D’Asseler Y, Vorster M, Gongxeka H, van de Wiele C. Tuberculous lymphadenitis:
FDG PET and CT findings in responsive and nonresponsive disease. Eur J Nucl Med Mol Imaging FDG PET and CT findings in responsive and nonresponsive disease.

2. Harris SH, Khan MA, Khan R, Haque F, Syed A, Ansari MM. Mammary tuberculosis: Analysis of thirty 12. Harris SH, Khan MA, Khan R, Haque F, Syed A, Ansari MM. Mammary tuberculosis: Analysis of thirt
eight patients. ANZ J Surg 2006;76(4):234-237. [http://dx.doi.org/10.1111/j.1445-2197.2006.03692.x] 3. Fellah L, Leconte I, Weynand B, Donnez J, Berlière M. Breast tuberculosis imaging. Fertil Ster 2006;86(2):460-461. [http://dx.doi.org/10.1016/j.fertnstert.2006.03.020]

4. Afridi SP, Memon A, Rehman SU, Baig N. Spectrum of breast tuberculosis. J Coll Physicians Surg Pak 2009;19(3):158-161. [http://dx.doi.org/03.2009/jcpsp.158161]

15. Ramaema DP, Buccimazza I, Hift RJ. Breast tuberculosis: Radiology spectrum with clinical correlationa retrospective analysis of 65 patients. ESR EPOS ${ }^{\mathrm{TM}}$. [http://dx.doi.org/10.1594/ecr2015/C-1154] 